\title{
Article \\ On the Parity of the Order of Appearance in the Fibonacci Sequence
}

\author{
Pavel Trojovský (1)
}

check for updates

Citation: Trojovský, P. On the Parity of the Order of Appearance in the Fibonacci Sequence. Mathematics 2021, 9, 1928. https://doi.org/10.3390/ math9161928

Academic Editor: Yang-Hui He

Received: 15 July 2021

Accepted: 12 August 2021

Published: 13 August 2021

Publisher's Note: MDPI stays neutral with regard to jurisdictional claims in published maps and institutional affiliations.

Copyright: (C) 2021 by the author. Licensee MDPI, Basel, Switzerland. This article is an open access article distributed under the terms and conditions of the Creative Commons Attribution (CC BY) license (https:/ / creativecommons.org/licenses/by/ $4.0 /)$.
Department of Mathematics, Faculty of Science, University of Hradec Králové,

50003 Hradec Králové, Czech Republic; pavel.trojovsky@uhk.cz; Tel.: +420-49-333-2860

Abstract: Let $\left(F_{n}\right)_{n \geq 0}$ be the Fibonacci sequence. The order of appearance function (in the Fibonacci sequence) $z: \mathbb{Z}_{\geq 1} \rightarrow \mathbb{Z}_{\geq 1}$ is defined as $z(n):=\min \left\{k \geq 1: F_{k} \equiv 0(\bmod n)\right\}$. In this paper, among other things, we prove that $z(n)$ is an even number for almost all positive integers $n$ (i.e., the set of such $n$ has natural density equal to 1 ).

Keywords: order of appearance; Fibonacci numbers; parity; natural density; prime numbers

\section{Introduction}

Let $\left(F_{n}\right)_{n}$ be the sequence of Fibonacci numbers which is defined by the recurrence $F_{n+2}=F_{n+1}+F_{n}$, with initial values $F_{0}=0$ and $F_{1}=1$. For any integer $n \geq 1$, the order of apparition (or the rank of appearance) of $n$ (in the Fibonacci sequence), denoted by $z(n)$, is the positive index of the smallest Fibonacci number which is a multiple of $n$ (more precisely, $z(n):=\min \left\{k \geq 1: n \mid F_{k}\right\}$ ). The arithmetic function $z: \mathbb{Z}_{\geq 1} \rightarrow \mathbb{Z}_{\geq 1}$ is well defined (as can be seen in Lucas [1], p. 300) and, in fact, $z(n) \leq 2 n$ is the sharpest upper bound (as can be seen in [2]). A few values of $z(n)$ (for $n \in[1,50]$ ) can be found in Table 1 (see the OEIS [3] sequence A001177 and for more facts on properties of $z(n)$ see, e.g., [4-9]).

Table 1. Values of $z(n)$ for $n$ from 1 to 50 .

\begin{tabular}{|c|c|c|c|c|c|c|c|c|c|}
\hline $\boldsymbol{n}$ & $\boldsymbol{z}(\boldsymbol{n})$ & $\boldsymbol{n}$ & $\boldsymbol{z}(\boldsymbol{n})$ & $\boldsymbol{n}$ & $\boldsymbol{z ( \boldsymbol { n } )}$ & $\boldsymbol{n}$ & $\boldsymbol{z}(\boldsymbol{n})$ & $\boldsymbol{n}$ & $\boldsymbol{z}(\boldsymbol{n})$ \\
\hline 1 & 1 & 11 & 10 & 21 & 8 & 31 & 30 & 41 & 20 \\
\hline 2 & 3 & 12 & 12 & 22 & 30 & 32 & 24 & 42 & 24 \\
\hline 3 & 4 & 13 & 7 & 23 & 24 & 33 & 20 & 43 & 44 \\
\hline 4 & 6 & 14 & 24 & 24 & 12 & 34 & 9 & 44 & 30 \\
\hline 5 & 5 & 15 & 20 & 25 & 25 & 35 & 40 & 45 & 60 \\
\hline 6 & 12 & 16 & 12 & 26 & 21 & 36 & 12 & 46 & 24 \\
\hline 7 & 8 & 17 & 9 & 27 & 36 & 37 & 19 & 47 & 16 \\
\hline 8 & 6 & 18 & 12 & 28 & 24 & 38 & 18 & 48 & 12 \\
\hline 9 & 12 & 19 & 18 & 29 & 14 & 39 & 28 & 49 & 56 \\
\hline 10 & 15 & 20 & 30 & 30 & 60 & 40 & 30 & 50 & 75 \\
\hline
\end{tabular}

Recall that the natural density of $\mathcal{A} \subseteq \mathbb{Z}_{>0}$ is the following limit (if it exists):

$$
\delta(\mathcal{A}):=\lim _{x \rightarrow \infty} \frac{\# \mathcal{A}(x)}{x},
$$

where $\mathcal{A}(x):=\mathcal{A} \cap[1, x]$ for $x>0$. In 2020, Trojovský [10] proved that natural density of the set $\{n \geq 1: z(n)<\epsilon n\}$ is 1 , for all $\epsilon>0$ (which generalized a result due to Marques [11] about $\lim \inf _{n \rightarrow \infty} z(n) / n$ ). Let $f: \mathbb{Z}_{\geq 1} \rightarrow \mathbb{Z}_{\geq 1}$ be an arithmetic function. We denote by $\mathcal{E}_{f}$ the set all $n$ for which $f(n)$ is an even number (i.e., $\left.\mathcal{E}_{f}:=\{n \geq 1: f(n) \equiv 0(\bmod 2)\}\right)$. For example, if $\phi(n)$ and $d(n)$ are the Euler totient function and the number-of-divisors 
function, respectively, then $\mathcal{E}_{\phi}=\mathbb{Z}_{\geq 3}$ and $\mathcal{E}_{d}=\mathbb{Z}_{\geq 1} \backslash\left\{n^{2}: n \geq 1\right\}$. In particular, both sets $\mathcal{E}_{\phi}$ and $\mathcal{E}_{d}$ have natural density equal to 1 . Another well-known arithmetic function related to Fibonacci numbers is the Pisano period $\pi: \mathbb{Z}_{>1} \rightarrow \mathbb{Z}_{>1}$ for which $\pi(n)$ is the smallest period of $\left(F_{k}(\bmod n)\right)_{k}$. The first few values of $\pi(n)($ for $n \in[1,50])$ are in Table 2 (see sequence A001175 in OEIS):

Table 2. Values of $\pi(n)$ for $n$ from 1 to 50.

\begin{tabular}{|c|c|c|c|c|c|c|c|c|c|}
\hline $\boldsymbol{n}$ & $\boldsymbol{\pi}(\boldsymbol{n})$ & $\boldsymbol{n}$ & $\boldsymbol{\pi}(\boldsymbol{n})$ & $\boldsymbol{n}$ & $\boldsymbol{\pi}(\boldsymbol{n})$ & $\boldsymbol{n}$ & $\boldsymbol{\pi}(\boldsymbol{n})$ & $\boldsymbol{n}$ & $\boldsymbol{\pi}(\boldsymbol{n})$ \\
\hline 1 & 1 & 11 & 10 & 21 & 16 & 31 & 30 & 41 & 40 \\
\hline 2 & 3 & 12 & 24 & 22 & 30 & 32 & 48 & 42 & 48 \\
\hline 3 & 8 & 13 & 28 & 23 & 48 & 33 & 40 & 43 & 88 \\
\hline 4 & 6 & 14 & 48 & 24 & 24 & 34 & 36 & 44 & 30 \\
\hline 5 & 20 & 15 & 40 & 25 & 100 & 35 & 80 & 45 & 120 \\
\hline 6 & 24 & 16 & 24 & 26 & 84 & 36 & 24 & 46 & 48 \\
\hline 7 & 16 & 17 & 36 & 27 & 72 & 37 & 76 & 47 & 32 \\
\hline 8 & 12 & 18 & 24 & 28 & 48 & 38 & 18 & 48 & 24 \\
\hline 9 & 24 & 19 & 18 & 29 & 14 & 39 & 56 & 49 & 112 \\
\hline 10 & 60 & 20 & 60 & 30 & 120 & 40 & 60 & 50 & 300 \\
\hline
\end{tabular}

Since $F_{i}=i$, for $i \in\{1,2\}$, the Pisano period may be defined as $\pi(n):=\min \{k \geq$ $1: F_{k} \equiv 0(\bmod n)$ and $\left.F_{k+1} \equiv 1(\bmod n)\right\}$. Thus, for $n \geq 3, \pi(n)$ is the order of the Fibonacci $Q$-matrix:

$$
Q=\left(\begin{array}{ll}
1 & 1 \\
1 & 0
\end{array}\right)
$$

in the general linear group $G L_{2}\left(\mathbb{Z}_{n}\right)$ (i.e., the invertible $2 \times 2$ matrices modulo $n$ ). In particular, $1=\operatorname{det}\left(Q^{\pi(n)}\right)=(\operatorname{det}(Q))^{\pi(n)}=(-1)^{\pi(n)}$, yielding that $\pi(n)$ is even, for all $n \geq 3$. In conclusion, $\mathcal{E}_{\pi}=\mathbb{Z}_{\geq 3}$.

Observe that $\pi(n)$ and $z(n)$ have similar definitions (in fact, these functions are strongly connected, as can be seen in Lemma 3$)$. However, they have very distinct behavior related to their parity. For example, $\mathbb{Z}_{\geq 1} \backslash \mathcal{E}_{z}$ is an infinite set (since $z\left(5^{k}\right)=5^{k}$ is an odd number, for all $k \geq 0$ ).

Therefore, the aim of this paper is to study some arithmetical and analytical properties of $\mathcal{E}_{z}:=\{n \geq 1: z(n) \equiv 0(\bmod 2)\}$. Our first result provides three infinite arithmetic progressions belonging to this set. More precisely,

Theorem 1. We have that $\{3 n, 4 n-1,4 n\} \subseteq \mathcal{E}_{z}$, for all $n \geq 1$.

Remark 1. We remark that the arithmetic progressions of Theorem 1 are the largest ones (in the sense of natural density) belonging to $\mathcal{E}_{z}$. In fact, for other arithmetic progressions of density of at least $1 / 4$, we have infinitely many terms which are outside of $\mathcal{E}_{z}$. Some of these terms can be made explicit. For example:

- If $n=5^{k}$, then $z(2 n)=3 \cdot 5^{k}$, for all $k \geq 0$.

- If $n=\left(5^{k}-1\right) / 2$, then $z(2 n+1)=5^{k}$, for all $k \geq 0$.

- If $n=\left(5^{2 k}-1\right) / 3$, then $z(3 n+1)=5^{2 k}$, for all $k \geq 0$.

- If $n=\left(5^{2 k+1}-2\right) / 3$, then $z(3 n+2)=5^{2 k+1}$, for all $k \geq 0$.

- If $n=\left(5^{k}-1\right) / 4$, then $z(4 n+1)=5^{k}$, for all $k \geq 0$.

- If $n=\left(5^{k}-1\right) / 2$, then $z(4 n+2)=3 \cdot 5^{k}$, for all $k \geq 0$.

We point out that Theorem 1 implies, in particular, that the natural density of $\mathcal{E}_{z}$ is at least $1 / 2$ (since $(4 n-1)_{n}$ and $(4 n)_{n}$ are disjoint arithmetic progressions with natural density equal to $1 / 4)$. In fact, it is possible to obtain a better lower bound for $\delta\left(\mathcal{E}_{z}\right)$, by noting that $\mathcal{E}_{z}$ also contains $(3 n)_{n}$ which has density $1 / 3$. Since the last sequence is not 
disjoint from the previous ones, we may use the inclusion-exclusion principle to deduce that the natural density of $\mathcal{E}_{z}$ is at least

$$
\frac{1}{4}+\frac{1}{4}+\frac{1}{3}-\frac{1}{12}-\frac{1}{12}=\frac{2}{3} \approx 0.66
$$

Table 3 suggests that for almost all positive integers $n$, one has that $z(n)$ is even (i.e., one expects that $\delta\left(\mathcal{E}_{z}\right)=1$ ). Note that a sieve method of searching by arithmetic progressions lying in $\mathcal{E}_{z}$ does not seem to reach the value 1.

Table 3. Quantity and proportion of arguments for which $z(n)$ is even.

\begin{tabular}{|c|c|c|c|c|c|c|c|c|}
\hline$x$ & 10 & 20 & 50 & 100 & 200 & 500 & 1000 & 3000 \\
\hline$\# \mathcal{E}_{z}(x)$ & 6 & 14 & 39 & 81 & 163 & 425 & 862 & 2632 \\
\hline$\frac{\# \mathcal{E}_{\mathcal{Z}}(x)}{x}$ & 0.6 & 0.7 & 0.78 & 0.81 & 0.815 & 0.85 & 0.862 & 0.877 \\
\hline
\end{tabular}

However, we were able to surmount this obstacle to confirm the expectation:

Theorem 2. There exists an effective computable constant $c>0$ such that

$$
\# \mathcal{E}_{z}(x) \geq x-\frac{c x}{(\log x)^{1 / 2}}
$$

for all $x>6.3 \times 10^{27}$. In particular, one has that $\delta\left(\mathcal{E}_{z}\right)=1$.

Remark 2. Note that all previously provided infinite sequences belonging to $\mathbb{Z}_{\geq 1} \backslash \mathcal{E}_{z}$ have exponential growth $\left(\right.$ e.g., $\left.\left(5^{n}\right)_{n}\right)$. In fact, the previous theorem ensures, in particular, that no infinite arithmetic progression can be within $\mathbb{Z}_{\geq 1} \backslash \mathcal{E}_{z}$ (since its natural density is 0 ).

The proof of the theorems combines Diophantine properties of $z(n)$ with analytical tools concerning primes in arithmetic progressions.

\section{Auxiliary Results}

In this section, we shall present some results which will be very important tools in the proof. The first ingredient is related to the value of $z\left(p^{k}\right)$, for a prime number $p$ and $k \geq 1$ :

Lemma 1 (Theorem 2.4 of [12]). We have that $z\left(2^{k}\right)=3 \cdot 2^{k-1}$, for all $k \geq 2$, and $z\left(3^{k}\right)=4 \cdot 3^{k-1}$, for all $k \geq 1$. In general, it holds that

$$
z\left(p^{k}\right)=p^{\max \{k-e(p), 0\}} z(p),
$$

where $e(p):=\max \left\{k \geq 0: p^{k} \mid F_{z(p)}\right\}$.

The next result provides some divisibility properties of the Pisano period for prime numbers.

Lemma 2 (Theorem 2.2 of [12]). Let $p$ be a prime number. We have that

(i) If $p \equiv \pm 1(\bmod 5)$, then $\pi(p)$ divides $p-1$.

(ii) If $p \equiv \pm 2(\bmod 5)$, then $\pi(p)$ divides $2(p+1)$. Furthermore, $\pi(p)=2(p+1) / t$, for some odd number $t$.

Observe that $F_{\pi(n)} \equiv F_{0} \equiv 0(\bmod n)$ and then $z(n)$ divides $\pi(n)$. Our next tool provides a characterization of the quotient $\pi(n) / z(n)$. In fact,

Lemma 3 (Theorem 1 of [13]). We have that $\pi(n) / z(n) \in\{1,2,4\}$, for all $n \geq 1$. Moreover, $\pi(n)=4 z(n)$ if and only if $z(n)$ is odd. 
The next tool is a kind of "formula" for $z(n)$ depending on $z\left(p^{a}\right)$ for all primes $p$ dividing $n$. The proof of this fact can be found in [14].

Lemma 4 (Theorem 3.3 of [14]). Let $n>1$ be an integer with prime factorization $n=p_{1}^{a_{1}} \cdots p_{k}^{a_{k}}$. Then

$$
z(n)=\operatorname{lcm}\left(z\left(p_{1}^{a_{1}}\right), \ldots, z\left(p_{k}^{a_{k}}\right)\right)
$$

In general, it holds that

$$
z\left(\operatorname{lcm}\left(m_{1}, \ldots, m_{k}\right)\right)=\operatorname{lcm}\left(z\left(m_{1}\right), \ldots, z\left(m_{k}\right)\right)
$$

In order to prove Theorem 2, we need an analytic tool related to the profusion of integers having factorization allowing only some classes of primes. The following notation will be used throughout this work: let $\mathbb{P}$ be the set of prime numbers and for an integer $q \geq 2$, set $\mathbb{P}(a, q)$ as the set of all prime numbers of the form $a+k q$, for some integer $k \geq 0$ (Dirichlet's theorem on arithmetic progressions) that ensures that $\mathbb{P}(a, q)$ is an infinite set whenever $\operatorname{gcd}(a, q)=1)$. Let $\mathcal{N}_{a, q}=\{n \geq 1: p \mid n \Rightarrow p \in \mathbb{P}(a, q)\}$ be the set of all positive integers whose prime factors lie exclusively in $\mathbb{P}(a, q)$. Additionally, denote $\beta:=1 / \phi(q)$ and

$$
G_{a, b}(s):=\zeta(s)^{-\beta} \prod_{p \in \mathbb{P}(a, q)}\left(1-p^{-s}\right)^{-1},
$$

which has an analytic continuation to a neighborhood of $s=1$. Here, as usual, $\zeta(s)$ denotes the Riemann zeta function.

Our last auxiliary lemma is a particular case of a theorem due to Chang and Martin [15]. We state this more precisely in the next lemma.

Lemma 5 (Theorem 3.4 of [15]). For any integer $q \geq 3$, there exists a positive absolute constant C such that uniformly for $q \leq(\log x)^{1 / 3}$, we have

$$
\#\left\{n \leq x: n \in \mathcal{N}_{a, q}\right\}=\frac{x}{(\log x)^{1-\beta}}\left(\frac{G_{a, q}(1)}{\Gamma(\beta)}+O\left(C(\log x)^{-1 / 4}\right)\right),
$$

where $\Gamma(z)=\int_{0}^{\infty} t^{z-1} e^{-t} d t$ is the Gamma function.

Now, we are ready to deal with the proof of the theorems.

\section{The Proofs}

\subsection{The Proof of Theorem 1}

The Case $z(3 n)$

Proof. First, in order to use Lemma 4, one may write $n=3^{k} m$, where $k \geq 0$ and $\operatorname{gcd}(m, 3)=1$. So, Lemma 4 yields that

$$
z(3 n)=z\left(3^{k+1} m\right)=\operatorname{lcm}\left(z\left(3^{k+1}\right), z(m)\right)=\operatorname{lcm}\left(4 \cdot 3^{k}, z(m)\right),
$$

where we used Lemma 1 . Hence we deduce that 4 divides $z(3 n)$.

The Case $z(4 n)$

Proof. Let us write $n=2^{k} m$, where $m$ is odd and $k \geq 0$. Thus, by Lemma 4 , we have

$$
z(4 n)=z\left(2^{k+2} m\right)=\operatorname{lcm}\left(z\left(2^{k+2}\right), z(m)\right)=\operatorname{lcm}\left(3 \cdot 2^{k+1}, z(m)\right),
$$

where we used Lemma 1 . Since $k+1 \geq 1$, then 2 divides $\operatorname{lcm}\left(3 \cdot 2^{k+1}, z(m)\right)=z(4 n)$, as desired. 
The Case $z(4 n+3)$

This case is more delicate, since $4 n+3$ is a prime number for infinitely many values of $n$. To overcome this difficulty, we deal first with these prime values.

Claim 1. If $p=4 k+3$ is a prime number, then $z(p)$ is even. The proof splits into two cases concerning the residues of $p$ modulo 5 . In fact, if $p \equiv \pm 1(\bmod 5)$, then Lemma 2 (i) implies that $\pi(p)$ divides $p-1$. Striving for a contradiction, suppose that $z(p)$ is odd. Then, by Lemma 3, we have that $\pi(p)=4 z(p)$ leading to the absurdity that $4 z(p)$ divides $p-1=4 n+2$. Thus, $z(p)$ is an even number. Now, for the case in which $p \equiv \pm 2(\bmod 5)$, one has, by Lemma 2 (ii), that $\pi(p)=2(p+1) / t$, for some odd integer $t$. Thus, by Lemma 3, we obtain

$$
2^{i} z(p)=\pi(p)=\frac{2(p+1)}{t}=\frac{8(n+1)}{t},
$$

for some $i \in\{0,1,2\}$. Thus, $z(p)=2^{3-i}(n+1) / t$ is an even number, since $3-i \geq 1$ and $t \equiv 1$ $(\bmod 2)$. This finishes the proof of the claim.

Proof. Let $4 n+3=p_{1}^{a_{1}} \cdots p_{k}^{a_{k}}$ be the prime factorization of the composite number $4 n+3$, with $a_{i} \geq 1$. By Lemma 4 , we obtain

$$
z(4 n+3)=\operatorname{lcm}\left(z\left(p_{1}^{a_{1}}\right), \ldots, z\left(p_{k}^{a_{k}}\right)\right) .
$$

Since the product of numbers $\equiv 1(\bmod 4)$ is also $\equiv 1(\bmod 4)$, then at least one of the prime factors of $4 n+3$, say $p_{1}$, must be of the form $4 \ell+3$. Therefore, by Lemma 1 , we can rewrite (2) as

$$
z(4 n+3)=\operatorname{lcm}\left(p_{1}^{\max \left\{a_{1}-e\left(p_{1}\right), 0\right\}} z\left(p_{1}\right), \ldots, z\left(p_{k}^{a_{k}}\right)\right) .
$$

However, $p_{1}=4 \ell+3$ is a prime number and the previous claim implies that $z\left(p_{1}\right)$ is even and so is $z(4 n+3)$ (since $z\left(p_{1}\right)$ divides $\left.z(4 n+3)\right)$. This completes the proof.

\subsection{The Proof of Theorem 2}

Proof. We have that

$$
\# \mathcal{E}_{z}(x)=\#\{n \leq x: z(n) \equiv 0 \quad(\bmod 2)\} .
$$

Note that since $z(n)$ is a multiple of $z(p)$, for all prime $p$ in the factorization of $n$ (by Lemma 4$)$, then a sufficient condition for $z(n)$ to be even is for $n$ to have some prime factor of the form $4 k+3$ (since $z(4 k+3)$ is even, by Theorem 1$)$. Therefore,

$$
\begin{aligned}
\# \mathcal{E}_{z}(x) & =\#\{n \leq x: z(n) \in 2 \mathbb{Z}\} \\
& \geq \#\{n \leq x: \exists p \mid n \text { with } p \equiv 3 \quad(\bmod 4)\} \\
& =x-\#\{n \leq x: p \mid n \Rightarrow p \equiv 1 \quad(\bmod 4)\} .
\end{aligned}
$$

Since

$$
\{n \leq x: p \mid n \Rightarrow p \equiv 1 \quad(\bmod 4)\}=\left\{n \leq x: x \in \mathcal{N}_{1,4}\right\} ，
$$

we can apply Lemma 5 in order to get an upper bound for the cardinality of the previous set. Thus, Lemma 5 (for $\beta=1 / \phi(4)=1 / 2$ ) implies the existence of a positive absolute constant $C$ such that

$$
\#\left\{n \leq x: n \in \mathcal{N}_{1,4}\right\}=\frac{x}{(\log x)^{1 / 2}}\left(\frac{G_{1,4}(1)}{\sqrt{\pi}}+O\left(C(\log x)^{-1 / 4}\right)\right),
$$

for all $x \geq e^{64} \approx 6.2 \times 10^{27}$ (where we used that $\Gamma(1 / 2)=\sqrt{\pi}$ ). Moreover, we have that 


$$
G_{1,4}(s):=\zeta(s)^{-1 / 2} \prod_{p \in \mathbb{P}(1,4)}\left(1-p^{-s}\right)^{-1}
$$

and

$$
G_{3,4}(s):=\zeta(s)^{-1 / 2} \prod_{p \in \mathbb{P}(3,4)}\left(1-p^{-s}\right)^{-1} .
$$

Since both sets $\mathbb{P}(1,4)$ and $\mathbb{P}(3,4)$ have the same density inside the set of all primes (namely, $1 / 2$ ), then $G_{1,4}(s) \asymp G_{3,4}(s)$ (i.e., $G_{1,4}(s) \ll G_{3,4}(s)$ and $G_{3,4}(s) \ll G_{1,4}(s)$ ). Now, we multiply (6) and (7) to arrive at

$$
\begin{aligned}
G_{1,4}(s) G_{3,4}(s) & =\zeta(s)^{-1}\left(\prod_{p \in \mathbb{P}(1,4)}\left(1-p^{-s}\right)^{-1}\right) \cdot\left(\prod_{p \in \mathbb{P}(3,4)}\left(1-p^{-s}\right)^{-1}\right) \\
& =\zeta(s)^{-1} \prod_{p \in \mathbb{P}(1,2)}\left(1-p^{-s}\right)^{-1} \\
& =\left(\prod_{p \in \mathbb{P}}\left(1-p^{-s}\right)\right) \cdot\left(\prod_{p \in \mathbb{P}(1,2)}\left(1-p^{-s}\right)^{-1}\right) \\
& =1-\frac{1}{2^{s}}
\end{aligned}
$$

where we used the Euler product $\zeta(s)=\prod_{p \in \mathbb{P}}\left(1-p^{-s}\right)^{-1}$ (see [16], p. 39) and that $\mathbb{P}(1,2)=\mathbb{P} \backslash\{2\}$. In particular, we have that $G_{1,4}(1) G_{3,4}(1)=1 / 2$ and so $G_{1,4}(1)=O(1)$. Therefore, there exists a positive constant $c$ such that (5) becomes

$$
\#\left\{n \leq x: n \in \mathcal{N}_{1,4}\right\} \leq \frac{c x}{(\log x)^{1 / 2}} .
$$

Finally, we deduce by combining (3), (4), and (8) that

$$
\# \mathcal{E}_{z}(x) \geq x-\frac{c x}{(\log x)^{1 / 2}}
$$

holds for all $x>6.3 \cdot 10^{27}>e^{64}$.

Additionally, since

$$
1 \geq \delta\left(\mathcal{E}_{z}\right)=\lim _{x \rightarrow \infty} \frac{\# \mathcal{E}_{z}(x)}{x} \geq \lim _{x \rightarrow \infty}\left(1-\frac{c}{(\log x)^{1 / 2}}\right)=1,
$$

we obtain that the natural density of $\mathcal{E}_{z}$ is equal to 1 . The proof is then complete.

\section{Further Comments}

We close this paper by offering one conjecture and two questions for further research. The natural generalization of the study presented in this work is to consider a similar problem by replacing 2 with another positive integer. For example, is $z(n)$ a multiple of 4 , for almost all positive integers $n$ ?

To study this problem, we start by defining (for an integer $m \geq 2$ ) $\mathcal{E}_{z}^{(m)}$ as the set of all $n \in \mathbb{Z}_{\geq 1}$ for which $z(n)$ is a multiple of $m$ (i.e., $\left.\mathcal{E}_{z}^{(m)}:=\{n \geq 1: z(n) \equiv 0(\bmod m)\}\right)$. In particular, $\mathcal{E}_{z}^{(2)}=\mathcal{E}_{z}$ and, by Theorem 2, one has that almost all positive integers belong to $\mathcal{E}_{z}^{(2)}$.

Note that, by Lemma $4, z(p)$ divides $z(m)$ whenever $p$ divides $m$. In particular,

$$
\# \mathcal{E}_{z}^{(z(p))}(x) \geq \frac{x}{p}
$$


yielding that the natural density of $\mathcal{E}_{z}^{(z(p))}$ is at least $1 / p$. In the case in which $p=3$, we infer that $\delta\left(\mathcal{E}_{z}^{(4)}\right) \geq 1 / 3$ (observe that $z(3)=4$ ). Moreover, we believe that

Conjecture 1. The natural density of $\mathcal{E}_{z}^{(4)}$ is equal to 1.

The main difficulty in proving the last statement is to find some infinite arithmetic progression (containing infinitely many prime numbers) lying completely in $\mathcal{E}_{z}^{(4)}$ (in the spirit of Theorem 1). In general, we conclude by posing the following questions:

Question 1. Are there infinitely many prime numbers p, for which $\delta\left(\mathcal{E}_{z}^{(p)}\right)=1$ ?

Question 2. Let $m \geq 1$ be an integer. Is it possible to provide an explicit positive lower bound for $\# \mathcal{E}_{z}^{(m)}(x)$ ?

\section{Conclusions}

In this paper, we study a Diophantine problem related to the arithmetic function $z: \mathbb{Z}_{\geq 1} \rightarrow \mathbb{Z}_{\geq 1}$, defined as $z(n):=\min \left\{k \geq 1: n \mid F_{k}\right\}$ (the so-called order of appearance in the Fibonacci sequence). The problem is to establish some properties of $\mathcal{E}_{z}:=\{n \geq$ $1: 2 \mid z(n)\}$. Indeed, we provide three infinite arithmetic progressions lying entirely within $\mathcal{E}_{z}$ (which implies, in particular, that the natural density of $\mathcal{E}_{z}$ is at least $2 / 3$ ). Furthermore, we provide a lower bound for $\#\left(\mathcal{E}_{z} \cap[1, x]\right)$ (for all sufficiently large $x$ ) which, in particular, confirms the expectation: the natural density of $\mathcal{E}_{z}$ is 1 . We close the work with some comments, questions, and a conjecture for further research. The proof combines arithmetical and analytical tools in number theory.

Funding: The research was supported by the Excellence Project PřF UHK No. 2213/2021-2022., University of Hradec Králové, Czech Republic.

Institutional Review Board Statement: Not applicable.

Informed Consent Statement: Not applicable.

Data Availability Statement: Not applicable.

Acknowledgments: The author thanks the University of Hradec Králové for support.

Conflicts of Interest: The author declares no conflict of interest.

\section{References}

1. Lucas, E. Théorie des fonctions numériques simplement périodiques. Am. J. Math. 1878, 1, 289-321. [CrossRef]

2. Sallé, H.J.A. Maximum value for the rank of apparition of integers in recursive sequences. Fibonacci Q. 1975, 13, 159-161.

3. Sloane, N.J.A. The On-Line Encyclopedia of Integer Sequences. Available online: http://www.research.att.com/ njas/sequences/ (accessed on 5 July 2021).

4. Marques, D. Fixed points of the order of appearance in the Fibonacci sequence. Fibonacci Q. 2012, 50, $346-352$.

5. Somer, L.; Krŕžžek, M. Fixed points and upper bounds for the rank of appearance in Lucas sequences. Fibonacci Q. 2013, 51, 291-306.

6. Sun, Z.H.; Sun, Z.W. Fibonacci numbers and Fermat's last theorem. Acta Arith. 1992, 60, 371-388. [CrossRef]

7. Luca, F; Tron, E. The distribution of self-Fibonacci divisors. In Advances in the Theory of Numbers; Fields Institute Communications; Springer: New York, NY, USA, 2015; Volume 77, pp. 149-158.

8. Trojovský, P. On Diophantine equations related to order of appearance in Fibonacci sequence. Mathematics 2019, 7, 1073. [CrossRef]

9. Trojovská, E. On the Diophantine Equation $z(n)=(2-1 / k) n$ Involving the Order of Appearance in the Fibonacci Sequence. Mathematics 2020, 8, 124. [CrossRef]

10. Trojovský, P. Some problems related to the growth of $z(n)$. Adv. Differ. Equ. 2020, 2020, 270. [CrossRef]

11. Marques, D. Sharper upper bounds for the order of appearance in the Fibonacci sequence. Fibonacci Q. 2013, 51, 233-238.

12. Fulton, J.D.; Morris, W.L. On arithmetical functions related to the Fibonacci numbers. Acta Arith. 1969, 16, 105-110. [CrossRef]

13. Vinson, J. The relation of the period modulo to the rank of apparition of $m$ in the Fibonacci sequence. Fibonacci Q. 1963, 1, 37-46.

14. Renault, M. Properties of the Fibonacci Sequence Under Various Moduli. Master's Thesis, Wake Forest University, Winston-Salem, NC, USA, 1996. Available online: http://webspace.ship.edu/msrenault/fibonacci/FibThesis.pdf (accessed on 3 August 2021). 
15. Chang B.; Martin, G. The smallest invariant factor of the multiplicative group. Int. J. Number Theory 2020, 16, 1377-1405. [CrossRef]

16. De Koninck, J.-M.; Luca, F. Analytic Number Theory-Exploring the Anatomy of Integers; American Mathematical Society: Providence, RI, USA, 2012. 